\title{
Relación de los índices de masa corporal y cintura-cadera con la capacidad residual funcional pulmonar en niños chilenos obesos versus normopeso: un estudio transversal
}

Relation among body mass index, waist-hip ratio, and pulmonary functional residual capacity in normal weight versus obese Chilean children: A cross-sectional study

\author{
Mg. Rodrigo Muñoz Cofréa,b, Dr. Mariano del Sola , Mg. Paul Medina González', \\ Lic. Javiera Escobar Inostroza ${ }^{d}$, Dr. Pablo A. Lizana ${ }^{e}$, Lic. Daniel Conei ${ }^{a, f} y$ \\ Mg. Máximo Escobar Cabello ${ }^{b, c}$
}

a. Programa de Doctorado en Ciencias Morfológicas, Universidad de La Frontera, Temuco, Chile.

b. Laboratorio de Función-Disfunción Ventilatoria.

c. Departamento de Kinesiología.

d. Programa de Magíster en Kinesiología. Universidad Católica del Maule, Talca, Chile.

e. Laboratory of Morphological Sciences, Instituto de Biología, Pontificia Universidad Católica de Valparaíso, Valparaíso, Chile.

f. Departamento de Ciencias Morfológicas, Facultad de Ciencia, Universidad San Sebastián, Puerto Montt, Chile.

Correspondencia: Mg. Rodrigo Muñoz Cofré: rodrigomunozcofre@ gmail.com

Financiamiento: Ninguno.

Conflicto de intereses: Ninguno que declarar.

Recibido: 22-6-2018 Aceptado: 12-12-2018

\section{RESUMEN}

Introducción. La obesidad está asociada a un descenso acelerado de la función ventilatoria. Las formas más frecuentes de evaluar el estado nutricional y medir la grasa abdominal y las caderas son el índice de masa corporal (IMC) y el índice cintura-cadera (ICC). Existe escasa evidencia que sugiera su relación con la capacidad residual funcional (CRF). Nuestro objetivo fue determinar la relación entre el IMC, el ICC y la CRF en niños obesos de la ciudad de Talca, Chile. Población y métodos. Se reclutaron niños de ambos sexos (6-12 años). Se evaluaron peso, talla, IMC, ICC y función pulmonar a través de pletismografía corporal. Dependiendo de la distribución de los datos, se utilizó la prueba $t$ de Student o U de Mann-Whitney para muestras independientes $\mathrm{y}$, la prueba $\mathrm{r}$ de Pearson o Spearman para establecer la correlación entre ICC y CRF.

Resultados. Los niños se dividieron en normopeso $(n=18)$ y obesos $(n=18)$. Se reportó una disminución significativa de la CRF $(p=0,025)$ en niños obesos y una relación inversa entre ICC y CRF, la cual fue moderada en niños normopeso $(s=-0,489 ; p=0,03)$ y alta en obesos $(\mathrm{r}=-0,681 ; \mathrm{p}=0,001)$.

Conclusiones. Los niños obesos mostraron una menor CRF respecto de los normopeso, que, a su vez, se relacionó con el ICC. Estos resultados indican efectos sistémicos que produce la obesidad en la función ventilatoria en niños y la necesidad de incorporar indicadores de distribución de grasa corporal a temprana edad. Palabras clave: índice de masa corporal, relación cintura-cadera, capacidad residual funcional, grasa abdominal.

http: / / dx.doi.org/10.5546/ aap.2019.230 Texto completo en inglés:

http: / / dx.doi.org/10.5546/ aap.2019.eng.230

Cómo citar: Muñoz Cofré R, del Sol M, Medina González P, Escobar Inostroza J, et al. Relación de los índices de masa corporal y cintura-cadera con la capacidad residual funcional pulmonar en niños chilenos obesos versus normopeso: un estudio transversal. Arch Argent Pediatr 2019;117(4):230-236.

\section{INTRODUCCIÓN}

La Organización Mundial de la Salud (OMS) define obesidad como "una acumulación anormal o excesiva de grasa que puede ser perjudicial para la salud". Esta se ha incrementado de forma alarmante en los países subdesarrollados. ${ }^{1}$ En Chile, la obesidad infantil ha continuado en aumento, en especial, desde 2009, después de la crisis económica. ${ }^{2,3}$ Los últimos datos disponibles del Ministerio de Salud (MINSAL) muestran que, en los menores de 6 años, la obesidad, en 2007, era de un 9,6\% y, en 2010, llegó a un 9,9\%.

La masa grasa es el componente más variable de la composición corporal, y existen riesgos asociados al exceso de esta y también a su localización. ${ }^{5}$ Es así como, en la actualidad, se ha comprobado que las consecuencias metabólicas y mecánicas de la distribución regional de la grasa tienen particular importancia en la evaluación clínica y en las conductas por seguir a partir de esta. ${ }^{6}$

Uno de los tantos problemas asociados a la obesidad infantojuvenil es el descenso acelerado de la función ventilatoria, y existen procesos mecánicos e inflamatorios de esta condición que disminuyen el rendimiento toracopulmonar ${ }^{7}$ y provocan cambios en la fisiología respiratoria, mecánica ventilatoria y músculos ventilatorios, ${ }^{8}$ lo que 
reduce la expansión torácica y el calibre de las vías aéreas. ${ }^{9}$ Así, la disminución de la capacidad residual funcional (CRF), volumen pulmonar al final de una respiración tranquila, es una alteración característica del síndrome de hipoventilación del obeso. ${ }^{10}$ Esta resulta del cambio en las presiones inspiratorias y espiratorias, debido al aumento de depósito de grasa en el tórax y en el abdomen. Además, el aumento de la presión intraabdominal es transmitida hacia el tórax, lo que genera cambios en la curva presión-volumen, con la consiguiente disminución de los volúmenes estáticos. ${ }^{6,9} \mathrm{Sin}$ embargo, existen pocos antecedentes sobre la repercusión de la localización del tejido graso y su relación con la CRF, considerando que este concepto, históricamente, se ha relacionado con problemas cardíacos. ${ }^{11}$

Se ha documentado la existencia de una relación inversa entre CRF e índice de masa corporal (IMC); ${ }^{6}$ este último es una medida global de la masa corporal que incluye la grasa y la masa magra, sin determinar las diferencias en la distribución del tejido graso, tanto en el tórax como en el abdomen. ${ }^{2}$ Esto es importante, debido a que la reducción de los volúmenes pulmonares en la obesidad es por el efecto mecánico de la grasa sobre la caja torácica, ya sea directa o indirecta. ${ }^{6,9}$ En este contexto, una de las formas más frecuentes de medir la grasa en el abdomen y en las caderas es el índice cinturacadera (ICC). Es una variable de distribución de tejido adiposo y es la más utilizada en la salud pública. Sin embargo, ha sido, principalmente, estudiada en asociación con factores de riesgo y enfermedades cardiovasculares. ${ }^{11}$ Se ha observado que existe una relación directa entre la grasa extra abdominal y la intraabdominal, ${ }^{8}$ hecho que provocaría desequilibrios en la respiración y alteraría la eficiencia del diafragma y, por tanto, los volúmenes pulmonares. ${ }^{6,9}$ No obstante, el ICC no ha sido considerado como indicador de problemas en la CRF de sujetos obesos. Así, este estudio contribuiría a demostrar que este índice podría predecir alteraciones respiratorias asociadas a la obesidad.

Considerando la información presentada, se hipotetiza que el aumento del IMC y del ICC se relacionarían con una disminución de la CRF en los niños obesos. El objetivo de esta investigación fue determinar la relación entre el IMC, el ICC y la CRF en los niños obesos de 6 a 12 años de la ciudad de Talca, Chile.

\section{POBLACIÓN Y MÉTODOS}

Investigación de corte transversal que se realizó entre los meses de marzo y mayo de 2017 en la ciudad de Talca. El tamaño de la muestra se calculó utilizando el software cálculo de tamaño muestral Ene 3,0 ${ }^{\circledR}$, (Barcelona, España).

A partir de un estudio piloto que contó con diez niños normopeso y diez obesos, determinando un nivel de significancia de 0,95, una potencia estadística del $80 \%$, un número de dos grupos, un porcentaje de abandono del $10 \%$ y una media de 2,19 y desviación estándar de 0,55 litros en CRF, se calculó un total de 36 niños de 6 a 12 años de la ciudad de Talca. ${ }^{12} \mathrm{La}$ población era de 220000 habitantes, de los cuales 30028 estaban en el rango de 5 a 14 años; a su vez, el $11,79 \%$ de este rango era obeso (http:/ / www. deis.cl/).

A través de un muestreo no probabilístico, se seleccionaron niños desde establecimientos educacionales. En una reunión con los apoderados, se presentó el proyecto y se registró a los padres que manifestaron su deseo de participar y, posteriormente, por vía telefónica, se les dio una hora para la evaluación. De acuerdo con su IMC, los niños se dividieron en dos grupos: normopeso, con IMC para la edad dentro de la desviación típica establecida por la mediana en los patrones de crecimiento infantil $(n=18)$, y obesos, con IMC para la edad con más de una desviación típica por encima de la mediana establecida en los patrones de crecimiento infantil de la $\mathrm{OMS}^{1}(\mathrm{n}=18)$. Las pruebas de función pulmonar se realizaron en el Laboratorio de Función y Disfunción Ventilatoria de la Universidad Católica del Maule por el kinesiólogo a cargo (certificado por el MINSAL). Para esto, el niño debió asistir acompañado de su apoderado. Los criterios de inclusión fueron encontrarse dentro del rango etario y ser normopeso $u$ obeso, y los de exclusión, tener incompatibilidad del estado físico y/o cognitivo, enfermedades respiratorias aguda o crónica, niñas que hubieran presentado menarquia, náuseas y vómitos. Esta investigación contó con la aprobación del Comité de Ética Científica de la Universidad Católica del Maule (Acta de Resolución 23/2016). La firma del consentimiento informado fue solicitada al padre o tutor legal $y$, antes de comenzar las evaluaciones, se le pidió el asentimiento a cada niño. Los resultados fueron entregados a los padres y se les indicó presentarlos en el siguiente control médico de su hijo. Por último, los datos obtenidos fueron estrictamente confidenciales y 
codificados paras resguardar la identidad de los participantes.

\section{Medidas antropométricas e indices}

Estatura: Se midió con un antropómetro SECA $^{\circledR}$ (modelo 220, Hamburgo, Alemania) la distancia desde el suelo al vértex. El sujeto debió estar de pie y descalzo, con los talones juntos y los pies formando un ángulo de $45^{\circ}$. Con los talones, los glúteos, la espalda y la región occipital en contacto con la superficie del antropómetro. La medición fue en inspiración máxima, manteniendo la cabeza en el plano meato-orbitario (de Frankfort). ${ }^{13}$ El valor fue registrado en metros.

Peso corporal: Se utilizó una balanza SECA ${ }^{\circledR}$ (modelo 840, Hamburgo, Alemania) para registrar el peso en kilogramos. ${ }^{13}$

IMC: Es un indicador de la relación entre el peso y la talla; se utilizó para identificar el sobrepeso y la obesidad. Se obtuvo dividiendo el peso en kilogramos por su talla al cuadrado en metros $\left(\mathrm{kg} / \mathrm{m}^{2}\right){ }^{1}$

Perímetro de cintura: El sujeto debió estar en posición de pie, relajado y con los brazos sobre el tórax. Se registró al final de una espiración normal el punto más estrecho entre la última costilla y en el margen de la cresta ilíaca o, en su defecto, en el punto medio si no había un punto de mayor estrechez. ${ }^{13}$ El valor fue registrado en centímetros.

Perímetro de cadera: El sujeto debió estar en posición relajada, de pie y con los miembros superiores plegados sobre el tórax. Se registró al final de una espiración normal la protuberancia máxima de los glúteos, que correspondió a la sínfisis del pubis. ${ }^{13}$

ICC: Es una medida antropométrica para determinar los niveles de grasa intraabdominal. Se calculó como la razón entre el perímetro de cintura y cadera. ${ }^{1}$

Volúmenes pulmonares: Las pruebas se realizaron en un pletismógrafo corporal Mediagraphics (Modelo Platinum Elite $D L^{\circledR}$ St. Paul, Minnesota, USA). Se midió según la normativa de la American Thorax Society (ATS). Con el sujeto sentado, se ajustó la pieza bucal a la altura de la cavidad oral con los labios bien cerrados durante la prueba para evitar fugas de aire, con pinza nasal y las manos en las mejillas para bloquear la musculatura facial. Posteriormente, se cerró la cabina y se indicó realizar cuatro respiraciones en volumen corriente. Se instruyó al sujeto para que "jadeara suavemente" intentando mover volúmenes de entre 50 y $60 \mathrm{ml}$ mientras bloqueaba las mejillas con los extremos de los dedos para evitar la fluctuación de la presión de la boca. La frecuencia de jadeo debió ser cercana a 60 por minuto $(1 \mathrm{~Hz})$. El profesional a cargo activó el obturador durante 2-3 segundos; después de esto, se indicó una inspiración máxima y, posteriormente, una espiración hasta el volumen residual (VR). ${ }^{14}$ Se seleccionó el mejor valor de tres maniobras con el $5 \%$ de variabilidad entre ellas en la capacidad pulmonar total (CPT). La CRF se presentó en litros.

Análisis estadístico: Se utilizó el programa estadístico GraphPad Prism (versión 5.0 $0^{\circledR}$, San Diego, USA). El manejo descriptivo de las variables fue mediante promedio \pm desviación estándar. Para observar las diferencias en el comportamiento del volumen de reserva espiratorio (VRE), el VR y la CRF según el estado nutricional de normopeso versus obeso, se utilizó la prueba $t$ de Student o U de MannWhitney, según correspondiera de acuerdo con la distribución de las variables. Para establecer la correlación entre el IMC, el ICC y la CRF, se efectuó la prueba $r$ de Pearson o Spearman, dependiendo de la distribución de los datos. Al respecto, se desarrollaron gráficas pertinentes según el estado nutricional. El nivel de significancia estadística se estableció en una $\mathrm{p}<0,05$.

\section{RESULTADOS}

De los 240 apoderados consultados, pertenecientes a seis cursos, se seleccionó a los primeros 18 niños normopeso y 18 obesos; estos fueron 15 de sexo femenino y 21 masculino, de entre 6 y 12 años. Las características de la muestra se observan en la Tabla 1. La comparación entre los grupos arrojó una diferencia significativa en las variables ICC, VRE y CRF entre los niños obesos y los normopeso (Tabla 1). Se observó una diferencia significativa de peso, IMC $(\mathrm{p}=0,0001)$ e ICC $(p=0,022)$ en los niños obesos, en relación con los normopeso; por el contrario, existió una diferencia significativa del VRE $(p=0,006)$ y la CRF $(p=0,025)$ de los niños obesos comparados con los normopeso (Figura 1). Además, se encontró una relación directa (moderada y significativa) entre el IMC y la CRF en los niños normopeso $(\mathrm{r}=0,681 ; \mathrm{p}=0,03)$ (Figura 2). Por último, existió una relación inversa entre el ICC y la CRF, la cual fue moderada en los niños normopeso $(s=-0,489 ; \mathrm{p}=0,03)$ y alta en los obesos $(\mathrm{r}=-0,681 ; \mathrm{p}=0,001)$ (Figura 3$)$. 
Tabla 1. Características antropométricas de los niños normopeso y los obesos de la comuna de Talca, Chile

\begin{tabular}{lccc}
\hline Variables & Normopeso & Obeso & Valor de $p$ \\
\hline Muestra total & 18 & 18 & - \\
Género (H-M) & $14 / 4$ & $7 / 11$ & - \\
Edad (años) & $10 \pm 2$ & $9 \pm 2$ & $0,257^{\mathrm{a}}$ \\
Peso (kg) & $34,36 \pm 6,39$ & $49,21 \pm 12,61$ & $0,0002^{\mathrm{b}}$ \\
Talla (m) & $1,41 \pm 0,11$ & $1,38 \pm 0,11$ & $0,759^{\mathrm{a}}$ \\
IMC (kg/m $)$ & $17,12 \pm 1,17$ & $25,50 \pm 3,26$ & $0,0001^{\mathrm{b}}$ \\
ICC & $0,86 \pm 0,08$ & $0,91 \pm 0,04$ & $0,022^{\mathrm{b}}$ \\
VRE (l) & $0,91 \pm 0,44$ & $0,60 \pm 0,30$ & $0,006^{\mathrm{b}}$ \\
VR (l) & $1,08 \pm 0,50$ & $0,81 \pm 0,47$ & $0,107^{\mathrm{b}}$ \\
CRF (l) & $1,99 \pm 0,67$ & $1,56 \pm 0,41$ & $0,025^{\mathrm{a}}$ \\
\hline
\end{tabular}

ICC: índice cintura-cadera; VRE: volumen de reserva espiratorio; VR: volumen residual; CRF: capacidad residual funcional; a: Mann-Whitney; b: t-Student.
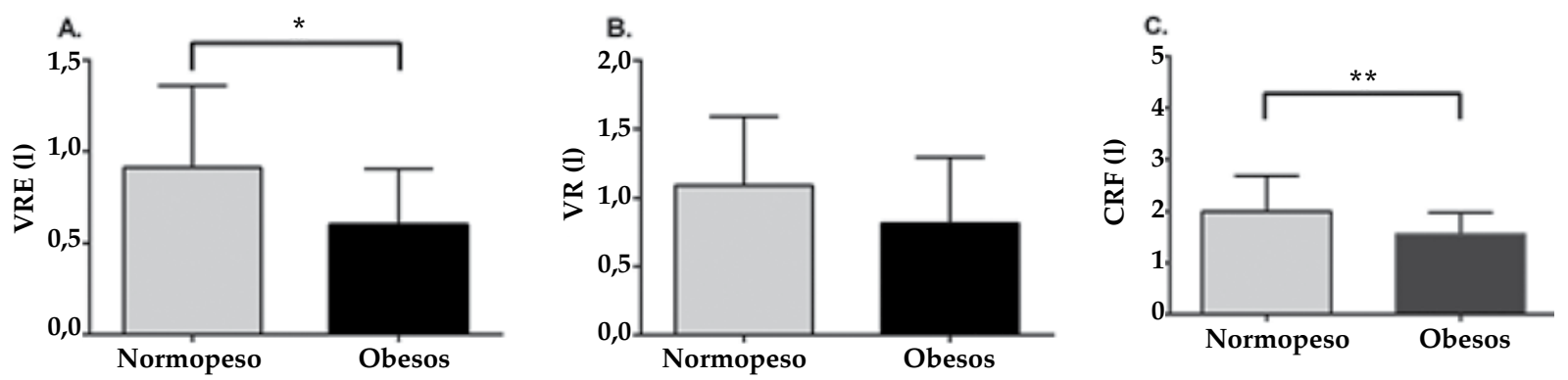

VRE: volumen de reserva espiratorio; VR: volumen residual; CRF: capacidad residual funcional.

A: comparación del VRE entre los niños normopeso y los obesos. Prueba estadística, Mann-Whitney;

B: comparación del VR entre los niños normopeso y los obesos. Prueba estadística, t-Student;

C: comparación de la CRF entre los niños normopeso y los obesos.

Prueba estadística, t-Student. ${ }^{*}=0,004 ;{ }^{* *}=0,03$.

FIGURA 2. Relación entre el indice de masa corporal y la capacidad residual funcional en los niños normopeso y los obesos de la comuna de Talca, Chile
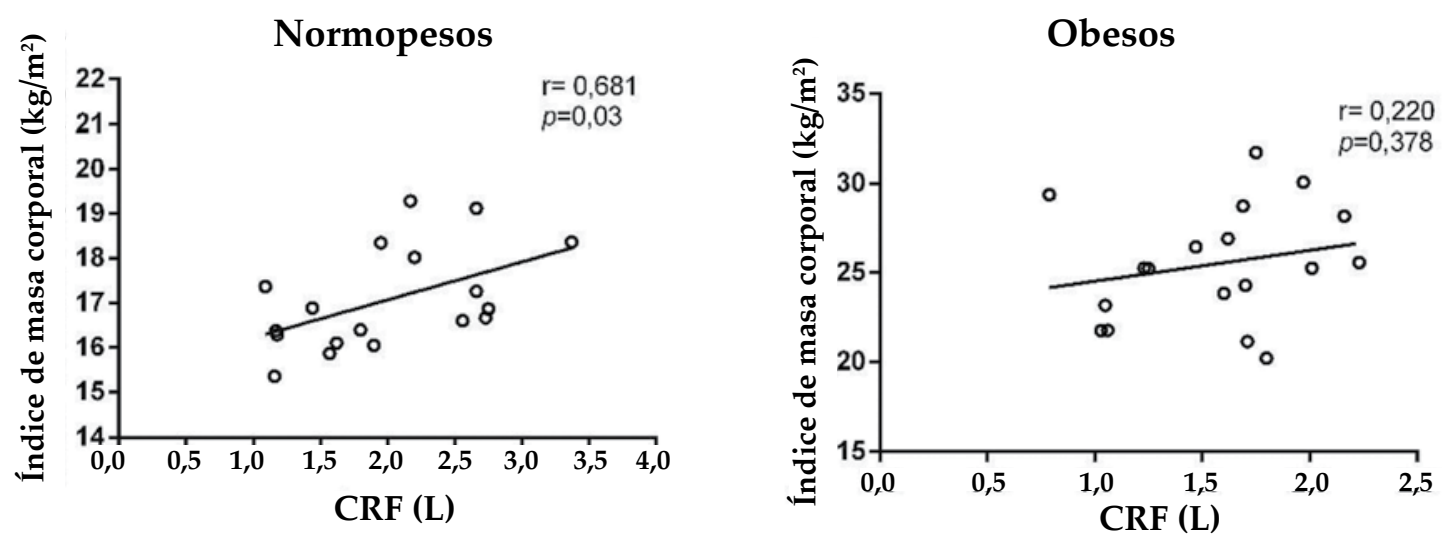

CRF: capacidad residual funcional; r: Pearson. 


\section{DISCUSIÓN}

El objetivo de esta investigación fue determinar la relación entre el IMC, el ICC y la CRF en los niños obesos de 6 a 12 años, de la ciudad de Talca, Chile. Los resultados indican que existió una baja significativa de la CRF en los niños obesos; así mismo, una relación inversa y alta entre el ICC y la CRF. La importancia de este hecho radica en detectar los efectos sistémicos que producen la obesidad y la distribución de grasa central en edades tempranas. Esto refuerza la utilidad del IMC e integra el ICC como indicador no solo cardiovascular, ${ }^{2,3}$ sino ventilatorio en los niños. ${ }^{11}$

Específicamente, la acumulación de tejido adiposo central disminuiría la elasticidad de la pared torácica, lo que reduciría la CRF y el VRE, y generaría una reducción prematura del flujo espiratorio durante una respiración en volumen corriente, en especial, en las bases pulmonares. Esto es reforzado por estudios que evidencian una relación inversa entre la CRF y la resistencia de las vías respiratorias en los sujetos obesos. ${ }^{6,9}$ Esto se sustenta en el modelo de tres compartimentos de Wilson, ${ }^{15}$ en el que la cavidad abdominal se considera asociada al tórax inferior $\mathrm{y}$, por tanto, puede afectar, positiva o negativamente, la actividad diafragmática a través de la pared del abdomen. ${ }^{16,17} \mathrm{El}$ rol de esta última sobre la bomba ventilatoria se ha descrito en modelos físicos como un agente pasivo que impactaría la contracción concéntrica del diafragma. Sin embargo, cuando este compartimento no se encuentra en condiciones normales, como, por ejemplo, en la obesidad, la mecánica ventilatoria se afectaría, lo que disminuiría, entre otras variables, la CRF.

Los resultados del presente estudio reafirman hallazgos previos en la población adulta y adulta mayor, en la que se determinó la asociación entre la función pulmonar y las variables antropométricas, ${ }^{15-18}$ específicamente, una disminución de la función pulmonar frente al aumento en el perímetro de cintura ${ }^{17}$ y el ICC, ${ }^{18-21}$ considerados un mejor predictor que el IMC. ${ }^{18,21}$ Ceylan et al. estudiaron la anormalidad predominante de la función pulmonar en los sujetos con sobrepeso y obesidad, y la correlación entre el deterioro de la función pulmonar y el grado de obesidad. Para esto, estudiaron a 31 mujeres y a 22 hombres con una edad media de 40 años. Encontraron una reducción en la CRF y en el VRE en los sujetos con sobrepeso y obesidad. Además, en las mujeres, el ICC y el IMC fueron mejores predictores para el VRE y, en los hombres, lo fue solamente el ICC. A partir de esto, concluyeron que los volúmenes pulmonares se veían influenciados por la distribución de la grasa corporal..$^{22}$ Esto concordó con nuestros resultados, en los que se evidenció una disminución significativa de la CRF y el VRE en los niños obesos, comparados con los normopeso. Es importante destacar que la presente muestra fue pediátrica y no se analizaron las diferencias entre los sexos.

La utilización del IMC en la caracterización de la función pulmonar ha sido discutida, ya que considera tanto la masa magra como la grasa, lo que complica determinar el efecto del compartimento abdominal sobre una

FIGURA 3. Relación entre el índice cintura-cadera y la capacidad residual funcional en los niños normopeso y los obesos de la comuna de Talca, Chile
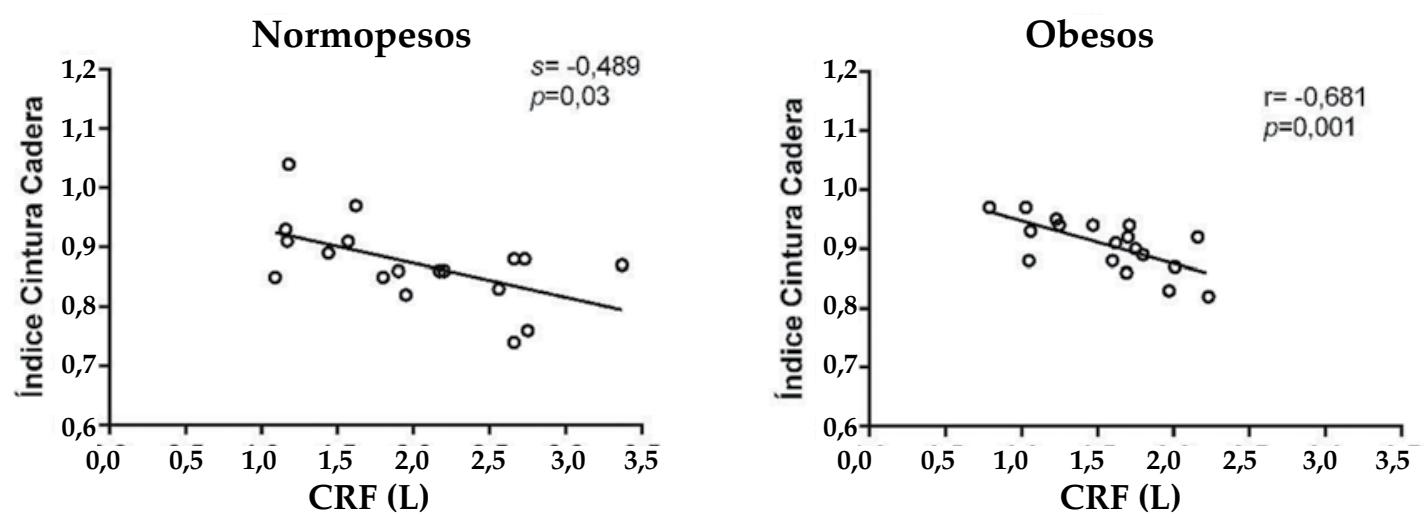

CRF: capacidad residual funcional; s: Spearman; r: Pearson. 
mecánica ventilatoria eficiente. ${ }^{21,23}$ Por ejemplo, al evaluar la función pulmonar mediante una espirometría en la población infantil obesa, según su IMC, ha demostrado tener resultados disímiles, y existe evidencia de una disminución de la capacidad vital forzada y el volumen espiratorio forzado dentro del primer segundo al aumentar el IMC. ${ }^{24}$ Además, no se han observado diferencias significativas, en las mismas variables, al comparar a los niños obesos con los normopeso. ${ }^{23,25}$

Los resultados obtenidos del VRE y de la CRF en los niños obesos demostraron que el IMC era sensible a los cambios de la función pulmonar en la muestra estudiada. Esto se debería a que, en pediatría, este índice presentaría una correlación positiva con el tejido adiposo y también se asociaría con factores de riesgo para el desarrollo de enfermedades crónicas no transmisibles. ${ }^{26} \mathrm{En}$ este contexto, el análisis asociado del IMC y del ICC sería lo más recomendable para determinar posibles trastornos del sistema ventilatorio. ${ }^{27}$ Cabe destacar que no se observaron diferencias en el VR; esto concordó con lo reportado en la literatura, en la que se indicó que esta variable se encontraba preservada. ${ }^{24}$

Además de esto, la muestra estudiada fue pediátrica y sin antecedentes de trastornos respiratorios, por tanto, se daría la ausencia de un deterioro del parénquima pulmonar, situación observada en los pacientes obstructivos crónicos adultos. En este último, el cierre prematuro de las vías aéreas de pequeño calibre da como resultado un aumento del VR y la limitación del flujo espiratorio, lo que, en su conjunto, produce un alza de la CRF. 12,28

Por último, no se debe olvidar que se evaluaron niños, que aún no habían alcanzado la madurez en su desarrollo físico y del sistema ventilatorio. Sumado a esto, la comprensión de la prueba por realizar variaba dependiendo de la etapa en la cual se encontraran, hechos que los diferenciaban de los adultos y que impactaban sobre el rendimiento en las pruebas de la función pulmonar. $^{29}$

\section{Limitaciones}

Este estudio contó con una serie de limitaciones. Como se mencionó anteriormente, la muestra evaluada no fue analiza según sexo, caso en que podrían existir correlaciones diferentes, debido a que no era parte de nuestro objetivo inicial. Futuros estudios deberían agregar esta variable con muestras de mayor tamaño. Debido a aspectos metodológicos y de cálculo muestral, los resultados reportados solo se aplicaron al grupo evaluado y no pueden ser generalizados. Sin embargo, los resultados concordaron con los de otros autores, por lo que fueron relevantes los datos encontrados en los niños obesos y su CRF.

\section{CONCLUSIONES}

Nuestros resultados indicaron que existió una disminución significativa de la CRF en los niños obesos, en comparación con los sujetos normopeso. Además, se reportó que, con mayor ICC, se reducía la CRF, lo que transformó el ICC en una importante herramienta para educadores físicos, kinesiólogos y profesionales de la salud como indicador de una posible disfunción ventilatoria en la edad pediátrica.

\section{REFERENCIAS}

1. Organización Mundial de la Salud. Sobrepeso y obesidad infantiles. [Consulta: 23 de febrero de 2018]. Disponible en: http: / / www.who.int/dietphysicalactivity/childhood/es/.

2. Lizana PA, Paula CV, Araya L, Aguilera F, et al. Obesity, Body Fat Distribution, and Physical Activity in School-age Children: an Urban and Rural Comparison in Valparaíso, Chile. Biomed Environ Sci. 2016; 29(11):834-39.

3. Lizana PA, González S, Lera L, Leyton B. Association between body composition, somatotype and socioeconomic status in Chilean children and adolescents at different school levels. J Biosoc Sci. 2018; 50(1):53-69.

4. Mönckeberg F, Muzzo S. La desconcertante epidemia de obesidad. Rev Chil Nutr. 2015; 42(1):96-102.

5. Xanthopoulos M, Tapia IE. Obesity and common respiratory diseases in children. Paediatr Respir Rev. 2017; 23:68-71.

6. Peters U, Suratt BT, Bates JHT, Dixon AE. Beyond BMI: Obesity and Lung Disease. Chest. 2018; 153(3):702-9.

7. Ogilvie RP, PatelSR. The epidemiology of sleep and obesity. Sleep Health. 2017; 3(5):383-8.

8. FengK, Chen L, HanS, Zhu GJ. Ratio of waist circumference to chest circumference is inversely associated with lung function in Chinese children and adolescents. Respirology. 2012; 17(7):1114-8.

9. Mafort TT, Rufino R, Costa CH, Lopes AJ. Obesity: systemic and pulmonary complications, biochemical abnormalities, and impairment of lung function. Multidiscip Respir Med. 2016; 11:28.

10. Lin CK, Lin CC. Work of breathing and respiratory drive in obesity. Respirology. 2012; 17(3):402-11.

11. Rosales R. Antropometría en el diagnóstico de pacientes obesos; una revisión. Nutr Hosp. 2012; 27(6):1803-9.

12. Parraguez Arévalo A, Rojas Navarro F, Ruz Céspedes M, Medina González P, et al. Influencia de la obesidad sobre la resistencia y conductancia específica de la vía aérea en niños escolares. Arch Argent Pediatr. 2018; 116(2):e227-33.

13. International Society for the Advancement of Kinanthropometry. International Standards for Anthropometric Assessment. Underdale: ISAK; 2001.

14. Wanger J, Clausen JL, Coates A, Pedersen OF, et al. Standardisation of the measurement of lung volumes. Eur Respir J. 2005; 26(3):511-22.

15. Wilson TA. Compartmental models of the chest wall and the origin of Hoover's sign. Respir Physiol Neurobiol. 2015; 210:23-9. 
16. De Troyer A, Boriek AM. Mechanics of the Respiratory Muscles. Compr Physiol. 2011; 1(3):1273-300.

17. Talasz H, Kremser C, Kofler M, Kalchschmid E, et al. Phase-locked parallel movement of diaphragm and pelvic floor during breathing and coughing-a dynamic MRI investigation in healthy females. Int Urogynecol J. 2011; 22(1):61-8

18. Santana H, Zoico E, Turcato E, Tosoni $\mathrm{P}$, et al. Relation between body composition, fat distribution, and lung function in elderly men. Am J Clin Nutr. 2001; 73(4):827-31.

19. Canoy D, Luben R, Welch A, Bingham S, et al. Abdominal Obesity and Respiratory Function in Men and Women in the EPIC-Norfolk Study, United Kingdom. Am J Epidemiol. 2004; 159(12):1140-9.

20. Chen Y, Rennie D, Cormier YF, Dosman J. Waist circumference is associated with pulmonary function in normal-weight, overweight, and obese subjects. Am J Clin Nutr. 2007; 85(1):35-9.

21. Sable M, Kowale AN, Aundhkar NV, Sable S. The Effect of Gender On The Relationship Between Body FatDistribution \& Lung Functions In Adult Indian Population. IOSR-JDMS. 2016; 15(3):73-7.

22. CeylanE, Cömlekçi A, Akkoçlu A, Ceylan C, etal. The effects of body fat distribution on pulmonary function tests in the overweight and obese. South Med J. 2009; 102(1):30-5.

23. Liyanage G, Jayamanne BD, Aaqiff M,Sriwardhana D. Effect of body mass index on pulmonary function in children. Ceylon Med J. 2016; 61(4):163-6.

24. Fretzayas A, Moustaki M, Loukou I, Douros K. Is obesity related to the lung function of non-asthmatic children? World J Clin Pediatr. 2018; 7(2):67-74.

25. Tenório LH, Santos AC, De Oliveira AS, De Lima AM, et al. Obesity and pulmonary function tests in children and adolescents: a systematic review. Rev Paul Pediatr. 2012; 30(3):423-30.

26. Boran $P$, Tokuc G, Pisgin B, Oktem S, et al. Impact of obesity on ventilatory function. J Pediatr (Rio J). 2007; 83(2):171-6.

27. Kaufer-Horwitz M, Toussaint G. Indicadores antropométricos para evaluar sobrepeso y obesidad en pediatría. Bol Med Hosp Infant Mex. 2008; 65(6):502-18.

28. Lufti MF. The physiological basis and clinical significance of lung volume measurements. Multidiscip Respir Med. 2017; 12:3.

29. Orliaguet G, Riou B, Leguen M. Maturation postnatale du diaphragme: des modifications ultrastructurales aux modifications fonctionelles. Ann Fr Anesth Reanim. 2004; 23(5):482-94.

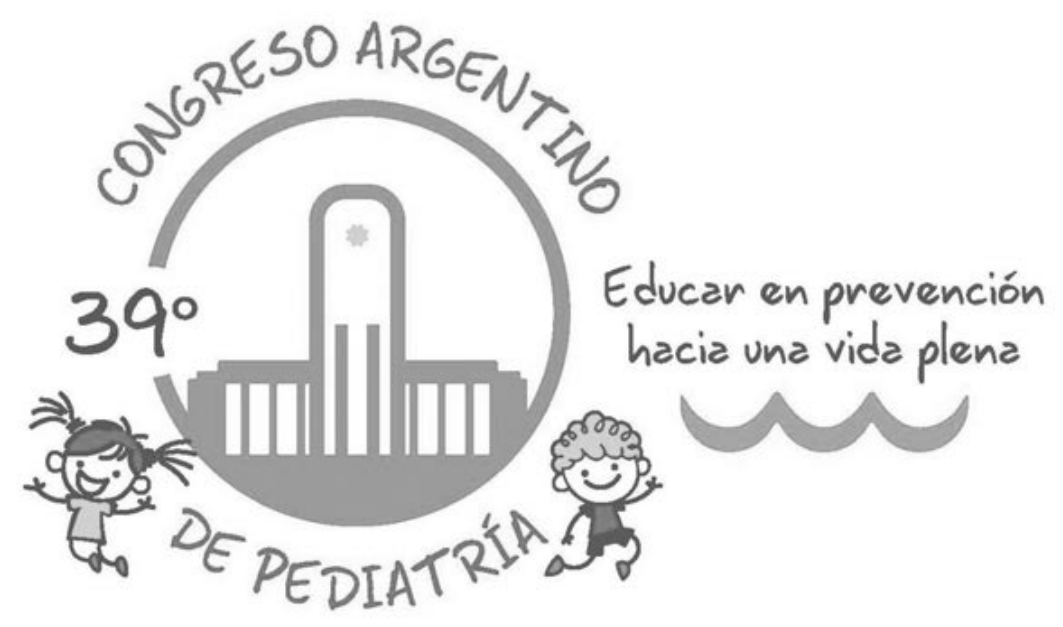

$39^{\circ}$ CONGRESO ARGENTINO
DE PEDIATRÍA
"Educar en prevención hacia una vida plena"
24, 25, 26 y 27 de septiembre de 2019

SEDE

Metropolitano Rosario

Junin 501 - Ciudad de Rosario - Provincia de Santa Fe

INFORMES E INSCRIPCIÓN

Sociedad Argentina de Pediatria - Entidad Matriz

Av. Coronel Díaz 1971 * (1425) Ciudad de Buenos Aires

Tel: (011)-4821-8612 / Fax: (011)-4821-8612 Interno 101

E-mail: congresos@sap.org.ar * Web Site: www.sap.org.ar 